\title{
Rigor mortis and livor mortis in a living patient: A fatal case of acute total occlusion of the infrarenal abdominal aorta following renal surgery
}

\author{
Christian Bjerre Høyer ${ }^{1, *}$, Leif Rognås², Lars Lund ${ }^{3,4}$, Lene Warner Thorup Boel ${ }^{1}$
}

'Section for Forensic Pathology and Clinical Forensic Medicine, Department of Forensic Medicine, Health, Aarhus University, Denmark ${ }^{2}$ Department of Anaesthesia, Aarhus University Hospital, Denmark

${ }^{3}$ Department of Urology, Odense University Hospital, Denmark

${ }^{4}$ University of Southern Denmark, Denmark

*Corresponding author. E-mail: cbh@dadlnet.dk

\begin{abstract}
:
A 63-year-old woman underwent a nephrectomy on the right side for renal cancer. Postoperatively she developed abdominal and lower back pain, which was treated with an injection of analgesics in an epidural catheter. The following morning it was discovered that the patient had cold legs with pallor and no palpable femoral pulse. Rigor mortis and livor mortis were diagnosed in both legs, even though the patient was still alive and awake. Doppler ultrasound examination revealed the absence of blood flow in the lower part of the abdominal aorta and distally. A cross disciplinary conference including specialists in urology, orthopaedics, vascular surgery, anaesthesiology, internal medicine, and intensive care concluded that no lifesaving treatment was possible, and the patient died the following day. A forensic autopsy revealed severe atherosclerosis with thrombosis and dissection of the abdominal aorta. This case clearly demonstrates that a vascular emergency should be considered when patients complain about pain in the lower back, abdomen or limbs. Clinicians should be especially aware of symptoms of tissue death that can be masked by epidural analgesia.

Keywords:

renal cancer, acute aortic occlusion, nephrectomy, signs of death, cause of death, rigor mortis, livor mortis
\end{abstract}

\section{INTRODUCTION}

Acute total occlusion of the infrarenal abdominal aorta is rare and usually described in case reports [1-7]. While rare, the severity of the situation is monumental, with mortality rates as high as 60-100 \% [5,8-13].

We present a case of acute total occlusion of the infrarenal abdominal aorta with a fatal outcome following nephrectomy.

\section{CASE}

A 63-year-old woman was admitted for elective right-sided nephrectomy, due to biopsy-proven renal cell carcinoma. The primary operation, performed several years earlier, was performed by laparoscopy without complications.

Her hereditary risk factors included a father who died of a cardiac attack and a sister and daughter diagnosed with coagulation defects. The patient had no history of thromboembolic episodes or cardiovascular disorders and did not receive medical treatment on a daily basis. It is explicitly stated in the hospital record that a pulse was bilaterally palpable in the dorsal foot artery.

Five thousand international units of dalterapin (Fragmin ${ }^{\circledR}$ Pfizer, New York, USA, a low molecular weight heparin) was administered as preoperative thrombosis prophylaxis. During surgery, bleeding from a minor arterial vessel could not be stopped, and the laparoscopic procedure was converted to an open procedure. After conversion, the bleeding artery was identified, and haemostasis was obtained. The right kidney was removed, and minor haemorrhages in the surrounding tissue were treated by the placement of haemostatic absorbable gauze (Surgicel ${ }^{\circledR}$, Ethicon, Somerville,
New Jersey, USA). The attending surgeon described the operation as "generally uncomplicated" in the hospital record.

The anaesthesia was uneventful with the exception of moderate hypertension at the end of the surgical procedure treated with bolus injections of fentanyl.

During the post-operative recovery, the patient experienced abdominal and lower back pain. Following medical examination, the pain was thought to be related to the surgical procedure and an anaesthesiologist inserted a thoracic epidural catheter without complications. A bolus injection of $3 \mathrm{ml}$ bupivacaine (Marcaine ${ }^{\oplus}$, AstraZeneca, London, United Kingdom) $5 \mathrm{mg} / \mathrm{ml}$ was followed by continuous infusion of bupivacaine (Marcaine ${ }^{\ominus}$ ) $2.5 \mathrm{mg} / \mathrm{ml}$. Intravenous analgesics were administered several times during the following hours due to recurrent abdominal pain, and the epidural catheter was retracted a few centimetres to provide better analgesia for the incision.

In the evening, the patient developed moderate hypotension. The administration of intravenous fluids and the continuous infusion of the $a_{1}$-receptor agonist phenylephrine hydrochloride (Metaoxedrin ${ }^{\oplus}$, Amgros, Copenhagen, Denmark) ensured circulatory stability, and the rest of the night was uneventful.

The following morning, a nurse discovered that the patient's legs were cold and pale and the femoral pulse was absent bilaterally. The attending consultant anaesthesiologist recognized rigor mortis and livor mortis in both legs, which was confirmed by repeat examination by other senior physicians. Bedside Doppler ultrasound examination by a radiologist revealed the total absence of distal blood flow and in the lower part of the abdominal aorta. 
At this point, the patient was aware and orientated in person, place and time, without pain and was circulatory stable.

A cross-disciplinary conference including specialists in urology, orthopaedics, vascular surgery, anaesthesiology, internal medicine, and intensive care concluded that no life-saving treatment was available. After receiving palliative care, the patient passed away the following morning.

The forensic autopsy revealed that the abdominal aorta was blocked four centimetres below the origin of the renal arteries by a four centimetrelong thrombus. Further, the infrarenal abdominal aorta was dissected from just below the origin of the renal arteries continuing bilaterally through the common iliac arteries and both external iliac arteries. Severe atherosclerosis was present throughout all the above-mentioned arteries (Figure 1).

An examination of the intestine showed a $12 \mathrm{~cm}$-long subperitoneal diffuse haemorrhage anal to the ileocaecal fold, continuing approximately five to six centimetres into the mesentery; in connection to this, a small artery was closed with a ligation clip. In addition, all internal organs supplied from the inferior mesenteric artery were normal. Other findings were ligation clips on the left renal vessels and the left ureter and haemostatic absorbable gauze (Surgicel ${ }^{\oplus}$ ).

The conclusion of the forensic autopsy was that the patient suffered from severe, asymptomatic and undiagnosed atherosclerosis and that the occlusion, as well as the dissection, was believed to be a complication of the overall manipulation of internal organs during the surgery. Thus, the manner of death was natural.

\section{DISCUSSION}

This case is unique, due to the extensive presence of rigor mortis and livor mortis in a person who was still alive.

The patient was examined by specialists in surgery, vascular surgery, orthopaedic surgery, urology and internal medicine. The diagnosis was made clinically and confirmed by colour-Doppler sonography, and intervention was not possible due to the presence of rigor mortis and livor mortis in the legs.

Acute total occlusion of the infrarenal abdominal aorta may immediately result in global ischaemia in the lowermost part of the body, a range of internal organs and both lower extremities, such as the common iliac arteries, and most probably, the inferior mesenteric artery will lose blood supply. Thus, organs such as the descending colon, the sigmoid colon, the rectum, the urinary bladder, the uterus and the ovaries or internal genitals, the gluteal muscles and both legs are endangered, although the collateral arterial blood supply from the superior mesenteric artery usually spares some parts of the organs [14].

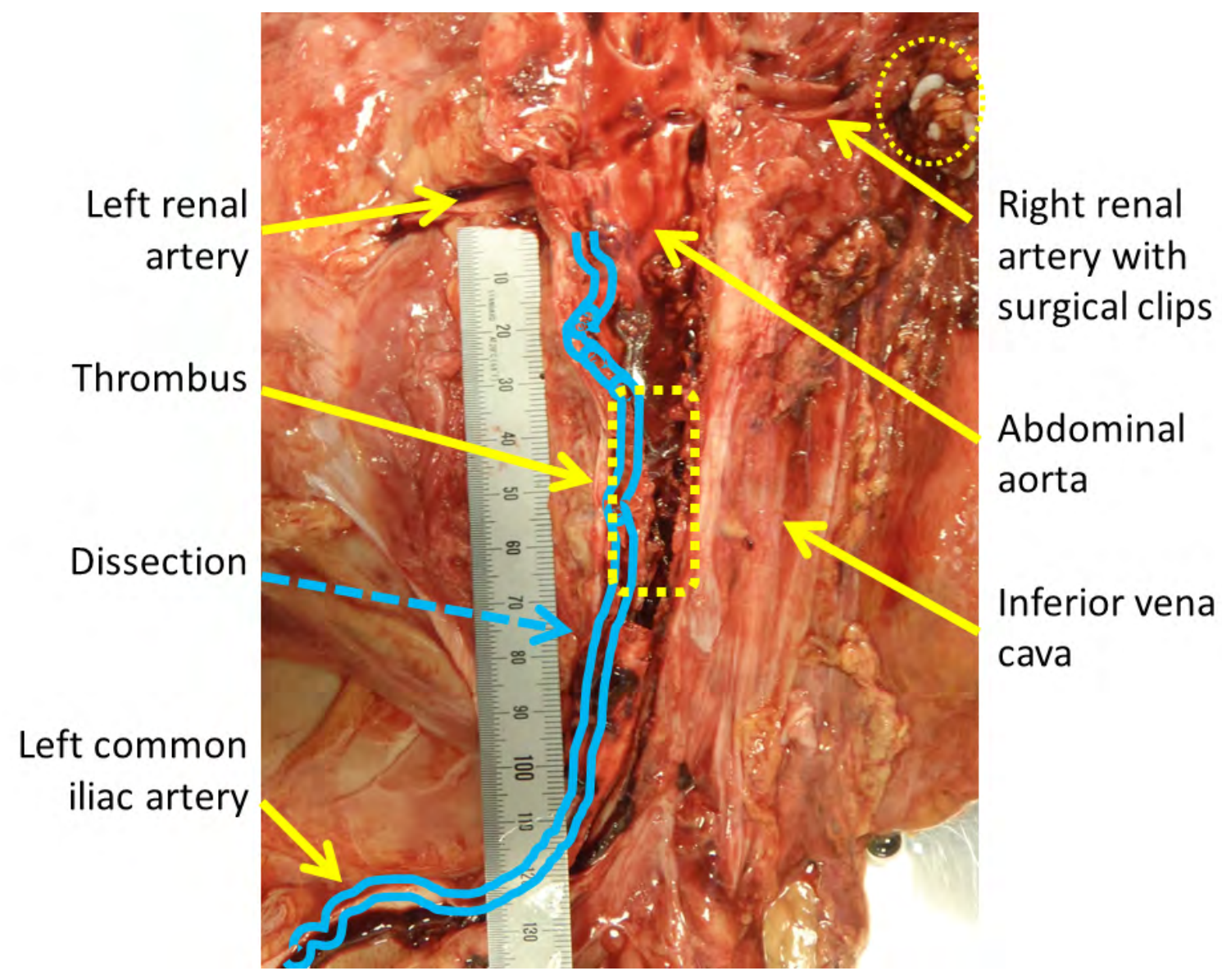

Figure 1. Organ block. The picture shows the posterior side of the organ block. Renal arteries are shown at the top (dotted circle highlights surgical clips). The abdominal aorta and the thrombus (dotted square) are shown, and the dissection of the infrarenal part of the abdominal aorta and the left common iliac artery are highlighted in blue. 
Major risk factors for acute aortic occlusion are coronary and peripheral vascular disease $[3,5,14]$, none of which were diagnosed in this patient.

\section{Diagnosis}

Classically, limb ischaemia is diagnosed by the "The Five P"-rule: pain, pallor, paralysis, paraesthesia, and pulselessness distal to the occlusion [15]. Progress of ischaemia is classified in viable (intact capillary refill, normal sensory and motor function, and the presence of arterial and venous Doppler signals), threatened (slow capillary refill slow, abnormal sensor and motor function, absent arterial Doppler signal, but present venous Doppler signal), and irreversible ischaemia leading to amputation or death (absent capillary refill, marked or absent sensory and motor function and absent arterial and venous Doppler signals) [15]

\section{Treatment}

Attempts at revascularization will most often lead to a fatal outcome [15]. This is due to the reperfusion syndrome: acute global ischaemia immediately initiates a wide range of unfavourable reactions in the tissue affected as anaerobic metabolism takes over [16,17]. A review of the reperfusion syndrome is not within the scope of this paper, but among the consequences of reperfusion are a global inflammatory response, pulmonary, renal, myocardial or hepatic failure and cardiac arrest due to flooding of the healthy part of the body with acidified blood with extreme concentrations of potassium (hyperkalaemia), lactic acid dehydrogenase, creatinine phosphokinase and myoglobin along with newly formed clots in the venous system of the ischaemic limb [18-20].

The presence of rigor mortis and livor mortis in a living person is quite spectacular in itself and to our knowledge is not sufficiently described in the literature. Heller et al. described the case of a hypothermic woman who apparently featured rigor mortis in the left arm and livor mortis in both the left arm and the left leg [21]. When found, respiration was insufficient and extreme bradycardia was present. However, as both phenomena disappeared upon warming and breathing and circulation were restored, the diagnosis couldn't have been rigor mortis and livor mortis. This highlights the fact that rigor mortis should be diagnosed with extreme caution in cases of hypothermia as it may be in fact "cold rigor". Chakravarthy reported a case of rigor mortis in a patient suffering from several severe circulatory diseases and insufficient peripheral circulation who developed severe circulatory complications during extensive cardiac surgery [22]. While deeply unconscious, the patient developed rigor mortis in all extremities and irreversible cardiac arrest.

Both cases [21,22] differ significantly from this case because our patient was awake and orientated when rigor mortis and livor mortis were diagnosed and widespread in both lower extremities. To our knowledge, the closest case described in the literature was presented by Triantafyllopoulos et al. who described a 56-year old man who developed flaccid paraplegia and livor mortis of both the lower extremities [20]. Thrombectomy and revascularization were performed, but the patient died within ten hours due to reperfusion syndrome. However, the lower limbs were found with flaccid paralysis, not regular rigor mortis.

The patients undergoing laparoscopic nephrectomy are handled in an accelerated manner/fast track and they do not routinely receive an epidural catheter because it is unnecessary. In the present case, due to the conversion and pain, it was decided to offer a thoracic epidural analgesia, which is widely used to provide adequate per- and post-operative analgesia for major abdominal surgery [23]. The insertion of an epidural catheter and the following continuous infusion of a local anaesthetic in this patient was according to the departmental standards as well as in concordance with international recommendations [23]. Further, pharmacological antithrombotic treatment is contraindicated for the six hours following the insertion of the epidural catheter.

In our case, diagnosis was delayed as three out of the five items

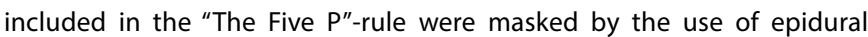
anaesthesia: pain, paralysis, and paraesthesia. Further, the patient wore compression stockings obstructing the direct observation of possible pallor. Thus, only one sign, pulselessness, was left to diagnose acute lower limb ischaemia. It can be argued that the emergence of symmetric pain in the lower limbs should have raised suspicion of the possibility of acute lower limb ischaemia. However, due to the low incidence of complications to renal surgery and the generally very low incidence of acute occlusion of the infrarenal abdominal aorta, we believe the complex characteristics of this case delayed the diagnosis. The patient's legs were not examined from the time the epidural catheter was inserted until the next morning. When found in the morning, rigor mortis and livor mortis were present in both lower extremities. This represents the advanced general extent of the processes following acute ischaemia. The level of the occlusion made amputation of all necrotizing tissue impossible, as this would not be compatible with life. The possibility of successful thrombolysis or thrombectomy was dismissible.

\section{CONCLUSION}

This case demonstrates the importance of performing a forensic autopsy in cases when patients die postoperatively. Establishing the cause of death as the progression of an existing disease and the manner of death as natural is legally important. Clinically, this information can be used to soothe the next of kin as well as the staff involved in this type of unfortunate case and can assure staff of their professionalism.

A postoperative evaluation of pulses in the lower limbs should be included when patients complain about pain in the lower back, abdomen or limbs. Likewise, clinicians should remember that bilateral paraesthesia and paralysis of the lower extremities may not be a neurological condition but also a vascular emergency. Finally, clinicians should be especially aware of the symptoms of tissue death that can be masked by epidural analgesia. The present case has led to the implementation of a new procedure ensuring that foot pulses are checked postoperatively.

\section{CONFLICTS OF INTEREST AND AUTHORS'CONTRIBUTIONS}

The authors declare that they have no conflict of interest.

The authors $\mathrm{CBH}, \mathrm{LL}, \mathrm{JBH}$, and LWTB have all contributed to the drafting and revision of the paper and the final version submitted for publication. The forensic autopsy was performed by authors CBH and LWTB, and the clinical evaluation of the patient during the postoperative course of events was performed by authors LL and LR. 


\section{REFERENCES}

[1] Cowan KN, Lawlor DK (2006) Sudden onset of paraplegia from acute aortic occlusion: a review of 2 cases and their unique presentation Am.J.Emerg.Med. 24: 479-81.

[2] Wong SS, Roche-Nagle G, Oreopoulos G (2013) Acute thrombosis of an abdominal aortic aneurysm presenting as cauda equina syndrome J.Vasc.Surg. 57: 218-20.

[3] Witz M, Lehmann J, Shnaker A, Korzets Z (2007) Acute occlusion of the abdominal aorta associated with lower limb paralysis Isr.Med.Assoc.J. 9: 115-6.

[4] Drager SB, Riles TS, Imparato AM (1979) Management of acute aortic occlusion Am.J.Surg. 138: 293-5.

[5] Surowiec SM, Isiklar H, Sreeram S, Weiss VJ, Lumsden AB (1998) Acute occlusion of the abdominal aorta Am.J.Surg. 176: 193-7.

[6] Dossa CD, Shepard AD, Reddy DJ, Jones CM, Elliott JP, Smith RF et al. (1994) Acute aortic occlusion. A 40-year experience Arch.Surg. 129: 603-7.

[7] Ketonen P, Harjola PT, la-Kulju K, Mattila T, Luosto R, Verkkala K (1986) Surgical treatment of occlusion of the infrarenal abdominal aorta. Experience with 55 patients Acta Chir Scand. 152: 665-8.

[8] Bell JW (1967) Acute thrombosis of the subrenal abdominal aorta Arch.Surg. 95: 681-4.

[9] Johnson JM, Gaspar MR, Movius HJ, Rosental JJ (1974) Sudden complete thrombosis of aortic and iliac aneurysms Arch.Surg. 108: 792-4.

[10] Littooy FN, Baker WH (1986) Acute aortic occlusion--a multifaceted catastrophe J.Vasc.Surg. 4: 211-6.

[11] Pietri P, Adovasio R, Pancrazio F, Sichel L, Fazio F (1987) Acute thrombosis of the infrarenal aorta. Therapeutic problems Int.Angiol. 6: 397-400.

[12] Webb KH, Jacocks MA (1988) Acute aortic occlusion Am.J.Surg. 155: 405-7.

[13] Babu SC, Shah PM, Nitahara J (1995) Acute aortic occlusion--factors that influence outcome J.Vasc.Surg. 21: 567-72.

[14] Hirose H, Takagi M, Hashiyada H, Miyagawa N, Yamada T, Tada S et al. (2000) Acute occlusion of an abdominal aortic aneurysm--case report and review of the literature Angiology 51:515-23.

[15] Meagher AP, Lord RS, Graham AR, Hill DA (1991) Acute aortic occlusion presenting with lower limb paralysis J.Cardiovasc.Surg.(Torino) 32: 643-7.

[16] Blaisdell FW (2002) The pathophysiology of skeletal muscle ischemia and the reperfusion syndrome: a review Cardiovasc.Surg. 10:620-30.

[17] Blaisdell FW (1989) The reperfusion syndrome Microcirc.Endothelium Lymphatics 5: $127-41$.

[18] Wilhelm MP, Schlensak C, Hoh A, Knipping L, Mangold G, Dallmeier Rojas D et al. (2005) Controlled reperfusion using a simplified perfusion system preserves function after acute and persistent limb ischemia: a preliminary study J.Vasc. Surg. 42: 690-4.

[19] Ali T, Castro J, Young CR, Burnand KG (2004) Complications of reperfusion in acute aortic artery occlusion following saddle embolization originating from an atrial myxoma Vascular. 12: 202-5.

[20] Triantafyllopoulos GK, Athanassacopoulos M, Maltezos C, Pneumaticos SG (2011) Acute infrarenal aortic thrombosis presenting with flaccid paraplegia Spine (Phila Pa 1976.) 36: E1042-E1045.

[21] Heller AR, Muller MP, Frank MD, Dressler J (2005) [Rigor mortis -- a definite sign of death?] Anasthesiol.Intensivmed.Notfallmed.Schmerzther. 40: 225-9.

[22] Chakravarthy M (2010) "Rigor mortis” in a live patient Am.J.Forensic Med.Pathol. 31: 87-8.

[23] Fawcett WJ, Baldini G (2015) Optimal analgesia during major open and laparoscopic abdominal surgery Anesthesiol.Clin. 33: 65-78. 